\title{
Comparing Clinical Profiles in Alzheimer's Disease and Parkinson's Disease Dementia
}

\author{
Martin R. Farlow ${ }^{\mathrm{a}}$ Frederick Schmitt $^{\mathrm{b}}$ Dag Aarsland ${ }^{\mathrm{c}, \mathrm{d}} \quad$ George T. Grossberg $^{\mathrm{e}}$ \\ Monique Somogyi ${ }^{f} \quad$ Xiangyi Meng ${ }^{f}$ \\ a Department of Neurology, Indiana University School of Medicine, Indianapolis, Ind., and \\ bSanders-Brown Center on Aging, University of Kentucky Medical Center, Lexington, Ky., USA; \\ 'Department of Neurobiology, Ward Sciences and Society, Alzheimer's Disease Research Center, \\ Karolinska Institutet, Stockholm, Sweden; ${ }^{d}$ Centre for Age-Related Diseases, Stavanger University

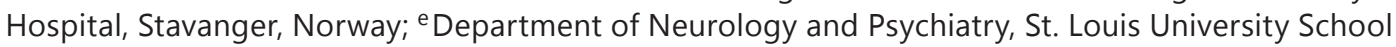 \\ of Medicine, St. Louis, Mo., and ${ }^{\mathrm{f}}$ Novartis Pharmaceuticals Corporation, East Hanover, N.J., USA
}

\section{Key Words}

Alzheimer's disease · Parkinson's disease dementia · Rivastigmine · Alzheimer's Disease Assessment Scale-cognitive subscale · Alzheimer's Disease Cooperative Study-Activities of Daily Living scale $\cdot 10$-item Neuropsychiatric Inventory · Alzheimer's Disease Cooperative Study-Clinical Global Impression of Change

\begin{abstract}
Background: Greater understanding of differences in baseline impairment and disease progression in patients with Alzheimer's disease (AD) and Parkinson's disease dementia (PDD) may improve the interpretation of drug effects and the design of future studies. Methods: This was a retrospective analysis of three randomized, double-blind rivastigmine databases (one in PDD, two in AD). Impairment on the Alzheimer's Disease Assessment Scale-cognitive subscale (ADAS-cog), Alzheimer's Disease Cooperative Study-Activities of Daily Living (ADCS$A D L)$ scale, 10-item Neuropsychiatric Inventory (NPI-10) and the ADCS-Clinical Global Impression of Change (CGIC) was compared [standardized difference (Cohen's d), similar if $<0.1$ ]. Results: Patients with AD or PDD had similar levels of impairment on the ADAS-cog and NPI10 . Scores on the ADCS-ADL scale (standardized difference $=0.47$ ) and the ADAS-cog memory domain (total, 0.33 ; items, $0.10-0.58$ ) were higher in AD; PDD patients were more impaired in the language $(0.23)$ and praxis $(0.34)$ domains. AD patients receiving placebo showed greater deterioration on the ADAS-cog (0.14) and improvement on the NPI-10 (0.11) compared with patients with PDD. Conclusion: Differing patterns of impairment occur in AD and PDD.
\end{abstract}

Some of the work described in the manuscript has previously been presented in poster format at the American Association for Geriatric Psychiatry (AAGP) Annual Meeting in San Antonio, Tex., March 18-21, 2011, and at the American Psychiatric Association (APA) Annual Meeting in Honolulu, Hawaii, May 14-16, 2011.

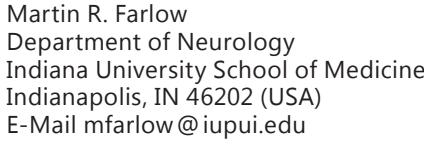




\section{Introduction}

Alzheimer's disease (AD) is a chronic, neurodegenerative disorder, a principle manifestation of which is loss of cholinergic transmission in the central nervous system [1]. Clinically, $\mathrm{AD}$ presents as progressive impairment in cognition, behavior and the patient's ability to perform activities of daily living (ADL).

A number of clinical trials have demonstrated that treatment with a cholinesterase inhibitor, such as rivastigmine, is associated with symptomatic improvements in cognition, behavior and the ability to perform ADL in patients with mild-to-moderate AD [2-4]. Rivastigmine is approved (in both oral and transdermal formulations) for the symptomatic treatment of mild-to-moderate AD in the USA and a number of other countries worldwide, and rivastigmine patch for severe AD in the USA.

Cholinergic system deficits are also pronounced in Parkinson's disease (PD) [5] and are believed to be associated with impaired cognitive function, leading to development of dementia in many patients with PD (Parkinson's disease dementia, PDD), especially as PD-related pathology begins to involve neocortical areas [6, 7]. Epidemiological studies have estimated that between 24 and $31 \%$ of patients with PD meet criteria for dementia [8]. Rivastigmine has demonstrated efficacy in mild-to-moderate PDD [9] and is approved for this indication in several countries, including the USA.

The Alzheimer's Disease Assessment Scale (ADAS) was developed to evaluate the severity and progression of both cognitive and non-cognitive dysfunction in patients with AD [10]. Validation of the cognitive subscale of the ADAS (ADAS-cog) has confirmed that the ADAS-cog can reliably assess increasing severity of dementia in patients with AD [11], and thus is now widely considered the gold standard measure of cognitive function in clinical drug trials of patients with mild-to-moderate AD. More recently, the use of the ADAS-cog in patients with PDD has also been validated [12]. The ADAS-cog assesses patients on 11 items, which evaluate three primary symptom domains: language, memory and praxis $[13,14]$.

Previous clinical studies of rivastigmine in AD and PDD provide a wealth of data pertaining to the use of rivastigmine in these patient populations $[2-4,9,15,16]$. More specifically, these databases contain large amounts of baseline and post-baseline ADAS-cog data, including individual item and symptom domain scores. In addition to cognitive assessments, clinical trials of rivastigmine in AD and PDD have also assessed efficacy on a number of other scales, including the Alzheimer's Disease Cooperative Study-Activities of Daily Living (ADCS-ADL) scale $[2,9,17]$, the 10-item Neuropsychiatric Inventory (NPI-10) $[2,9,18]$ and the Alzheimer's Disease Cooperative Study-Clinical Global Impression of Change (ADCS-CGIC) $[2-4,9,15,16$, 19]. Due to the large numbers of patients included in these databases, comparative analysis of the severity of baseline impairment on these measures across these trial populations may further elucidate disease-specific differences in patterns of impairment in these patients.

In a previous study, significantly different cognitive profiles were reported between carefully matched subjects with PDD and AD ( $n=488$ in each group) [20]. These groups differed significantly on all measures, except for the ADAS-cog item naming objects/fingers. Based on standardized Z-scores, the groups were shown to differ most in the categories attention and calculation taken from the Mini-Mental State Examination (MMSE; serial 7s subtraction task), followed by the ADAS-cog items orientation, praxis and following commands [20].

The aim of this retrospective, post hoc analysis was to use available clinical data $[2-4,9$, $15,16]$ to investigate whether there are differences in the range of impairments observed at baseline and the rates of change in these domains over time in AD and PDD patients. Baseline scores on the total ADAS-cog, total ADCS-ADL, total NPI-10, the ADAS-cog's symptom domains (language, memory and praxis), and the 11 individual ADAS-cog items were compared between the $\mathrm{AD}$ and PDD patient populations. Besides investigating baseline impairment, 
calculating the change from baseline to the study endpoint in the placebo groups provided longitudinal data on disease progression over time in untreated patients with AD or PDD. Deterioration in cognition (ADAS-cog), behavior (NPI-10), ADL (ADCS-ADL) and global function (ADCS-CGIC) was compared between patients with AD or PDD randomized to receive placebo in these controlled clinical studies. Data were provided by a much larger set of AD patients than have been considered in previous analyses.

\section{Materials and Methods}

Clinical Studies

A retrospective, exploratory analysis was conducted of three trial databases, namely (a) the Investigation of transDermal Exelon in ALzheimer's disease (IDEAL) trial, CENA713D2320; (b) the EXelon in PaRkinson's disEaSe dementia Study (EXPRESS), CENA713B2311, and (c) the Alzheimer's Disease with ENA 713 (ADENA) databases. The full details of these trial databases have been published previously $[2-4,9,15,16]$. Each of these studies was conducted according to the ethical principles of the Declaration of Helsinki.

Briefly, IDEAL was aninternational, 24-week, double-blind, placebo-and active-controlled trial designed to investigate the efficacy and safety of rivastigmine (patch and capsules) versus placebo in mild-to-moderate AD [2]. Eligible patients had a diagnosis of dementia of the Alzheimer's type [21,22] and probable AD of mild-to-moderate severity (MMSE score of $10-20$, inclusive) [23]. Patients were randomized to receive a rivastigmine patch (9.5 or 17.4 $\mathrm{mg} / 24 \mathrm{~h}$ ), rivastigmine capsules (12 mg/day) or placebo. Primary outcomes were the change from baseline at week 24 on the ADAS-cog and ADCS-CGIC. The ADCS-ADL and NPI were secondary outcomes.

EXPRESS was an international, 24-week, double-blind, placebo-controlled trial of rivastigmine in PDD [9]. Patients had a diagnosis of probable PD (UK Parkinson's Disease Society Brain Bank Clinical Criteria) and mild-to-moderately severe dementia (DSM-IV criteria; MMSE score of 10-24, inclusive). Study participants were randomized in a 2:1 ratio to receive rivastigmine capsules (3-12 $\mathrm{mg}$ /day) or placebo. Primary outcomes were the ADCS-CGIC and the change from baseline at week 24 on the ADAS-cog. The change from baseline at week 24 on the ADCS-ADL and NPI-10 were secondary outcome measures.

The ADENA program was a retrospective analysis of four 26-week, randomized, doubleblind, placebo-controlled trials of rivastigmine capsules in patients with mild-to-moderate $\operatorname{AD}[3,4,15,16]$. In all four trials, patients had been diagnosed with dementia of the Alzheimer's type (DSM-IV, NINCDS/ADRDA criteria) [21, 22] and probable AD of mild-to-moderate severity (MMSE scores of 10-26, inclusive) [23]. Patients were randomized to receive either rivastigmine capsules or placebo. Two studies evaluated rivastigmine 1-4 and 6-12 mg/day, one evaluated rivastigmine $2-12 \mathrm{mg} /$ day twice daily and thrice daily and the fourth evaluated rivastigmine 3, 6 and $9 \mathrm{mg} /$ day. Outcome measures in each of these studies included the change from baseline at week 26 on the ADAS-cog and the Clinician Interview-Based Impression of Change incorporating caregiver information (CIBIC-plus).

\section{Statistical Analysis}

In the current analysis, baseline ADAS-cog total and domain scores from the IDEAL and ADENA trial databases provided data on AD patients, and EXPRESS provided data on PDD patients. ADCS-ADL and NPI-10 data were available from the IDEAL and EXPRESS databases; however, these scales were not outcome measures in the ADENA program. ADCS-CGIC data were available from the IDEAL and EXPRESS patient populations, and CIBIC-plus data from 
Table 1. Baseline demographics and patient characteristics from the AD (ADENA and IDEAL) and PDD (EXPRESS) trial databases (randomized population)

\begin{tabular}{lllc}
\hline & $\begin{array}{l}\text { AD (ADENA and IDEAL) } \\
(\mathrm{N}=3,999)\end{array}$ & $\begin{array}{l}\text { PDD (EXPRESS) } \\
(\mathrm{N}=541)\end{array}$ & p value \\
\hline Mean age \pm SD, years & $73.2 \pm 8.04$ & $72.7 \pm 6.61$ & 0.1432 \\
Male:female, \% & $38.91: 61.09$ & $64.88: 35.12$ & $<0.0001^{*}$ \\
Ethnic origin, \% & & & $<0.0001^{*}$ \\
$\quad$ Caucasian & 89.02 & 99.63 & \\
Black & 2.68 & 0.00 & \\
Oriental & 2.95 & 0.00 & \\
$\quad$ Other & 5.35 & 0.37 & 0.0007 \\
MMSE score & 3,987 & 541 & $19.3 \pm 3.91$ \\
$\quad$ Patients, $\mathrm{n}$ & $18.7 \pm 4.36$ & & \\
Mean \pm SD &
\end{tabular}

* Where indicated, the $\mathrm{p}$ values were estimated using the $\chi^{2}$ test. All other $\mathrm{p}$ values derived from a two-sample t test.

ADENA were also included in the analysis. For each outcome measure, all patients who completed a baseline assessment were also included in the analyses. ADCS-CGIC scores and the mean change from baseline at week 24/26 on the ADCS-ADL, NPI-10 and ADAS-cog total and domain scores were calculated for the placebo group (intent-to-treat population).

The mean baseline score on the ADAS-cog, NPI-10 scale and ADCS-ADL scale and the standard deviation (SD) from the mean were calculated in the AD and PDD patients using an analysis of covariance (ANCOVA) model with study as a factor and baseline score as a covariate. The mean (SD) change from baseline at the study endpoint on each of the outcome measures was calculated using two ANCOVA models: model 1 included study as a factor and baseline score as a covariate (where appropriate), and model 2 included study as a factor and baseline MMSE score and corresponding baseline score as covariates (where appropriate). Standardized differences (Cohen's d) were calculated to compare baseline and change-frombaseline (where applicable) NPI-10, ADCS-ADL, ADCS-CGIC and ADAS-cog total, domain and item scores between the AD and PDD patient populations. Scores on each outcome measure were considered to be similar in the AD and PDD population, if the calculated standardized difference was below the applied threshold of 0.1.

\section{Results}

Patients

In total, baseline data were provided by 3,999 AD patients (from the ADENA and IDEAL databases) and 541 PDD patients (EXPRESS database). Baseline demographics and patient characteristics are summarized in table 1 . The AD and PDD patient populations were comparable in terms of age. However, the AD and PDD patient populations were significantly different in terms of gender, ethnic origin and their baseline MMSE score. The PDD population was predominantly male $(64.88 \%)$, while the AD population was predominantly female (61.09\%). Overall, $99.63 \%$ of the PDD population and $89.02 \%$ of the AD population were Caucasian. The PDD population tended to show a higher mean score at baseline on the MMSE than the AD population (table 1). 
Fig. 1. Baseline scores on the ADAS-cog symptom domains of language, memory and praxis for the AD (IDEAL and ADENA) and PDD (EXPRESS) patient populations (randomized population). Values represent calculated standardized differences comparing the AD and PDD patient populations. Error bars represent the SD from the mean. Standardized differences were calculated using an ANCOVA model with study as a factor and corresponding baseline score as a covariate (where appropriate). Higher scores reflect a greater impairment.

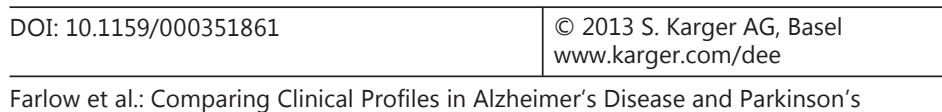

Farlow et al.: Comparing Clinical Profiles in Alzheimer's Disease and Parkinson's Disease Dementia

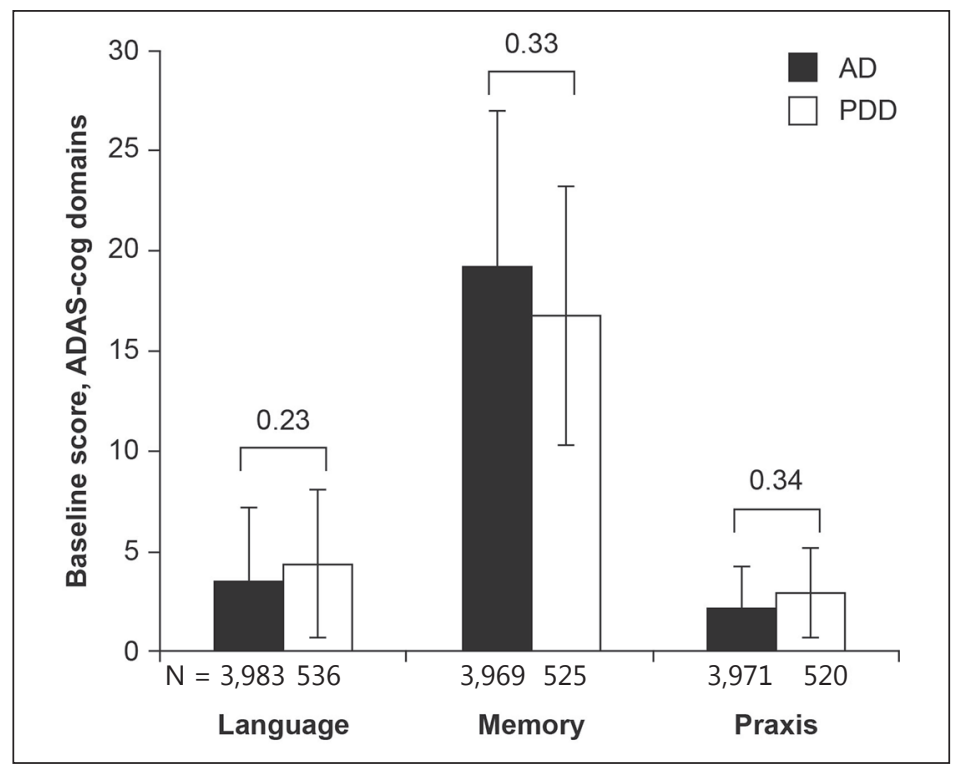

Comparison of Baseline ADAS-cog, ADCS-ADL and NPI-10 Data between the AD and PDD Patient Populations

The mean total baseline ADAS-cog score differed by one point between the AD and PDD populations, with $\mathrm{AD}$ patients showing a higher mean score. However, the calculated standardized difference of 0.09 falls below the applied threshold of 0.1 , suggesting that, overall, patients with PDD and AD had similar levels of cognitive impairment at baseline. The mean (SD) baseline ADCS-ADL scores were higher in the AD population than in the PDD population [AD: 49.25 (15.93), $\mathrm{N}=1$ 1,104; PDD: 41.46 (18.31), $\mathrm{N}=$ 498; standardized difference = 0.47]. In the PDD population, a significant correlation was observed between the baseline ADCS-ADL score and the baseline score on the Unified Parkinson's Disease Rating Scale Part V ( $\mathrm{r}=-0.40$; $\mathrm{p}<0.0001$ ), with the most severely affected patients showing the greatest impairment of ADL. Despite a trend towards higher baseline scores on the NPI-10 in patients with PDD compared with AD [PDD: 12.83 (12.16), $\mathrm{N}=500$; AD: 12.03 (12.16), $\mathrm{N}=1$ 103], the standardized difference of 0.07 suggests that, overall, behavioral disturbances manifested in similar levels in both the AD and PDD patients.

When considering the three domains of the ADAS-cog (language, memory and praxis), patients with AD showed greater baseline impairment on the ADAS-cog memory domain than patients with PDD (standardized difference $=0.33$; fig. 1). In contrast, PDD patients were more impaired than the $\mathrm{AD}$ patients on the language and praxis ADAS-cog domains (standardized difference $=0.23$ and 0.34 , respectively; fig. 1 ). AD patients were more impaired than PDD patients on all of the individual items (word recognition, naming objects/fingers, orientation, word recall and remembering test instructions) that comprise the ADAS-cog memory domain (fig. 2; standardized difference $=0.10-0.58$ ).

However, PDD patients showed greater impairment than AD patients on all items that comprise the ADAS-cog language domain (standardized difference $=0.14-0.39$ ), except for word-finding difficulty (standardized difference $=0.04$ ), and both items that comprise the ADAS-cog praxis domain (constructional praxis, standardized difference $=0.34$ and ideational praxis, standardized difference $=0.27$; fig. 2 ). Overall, the greatest difference in the severity of cognitive impairment between AD and PDD patients was seen on the orientation item, which forms part of the memory domain. Here, AD patients showed greater baseline impairment than PDD patients (standardized difference $=0.58$ ). 


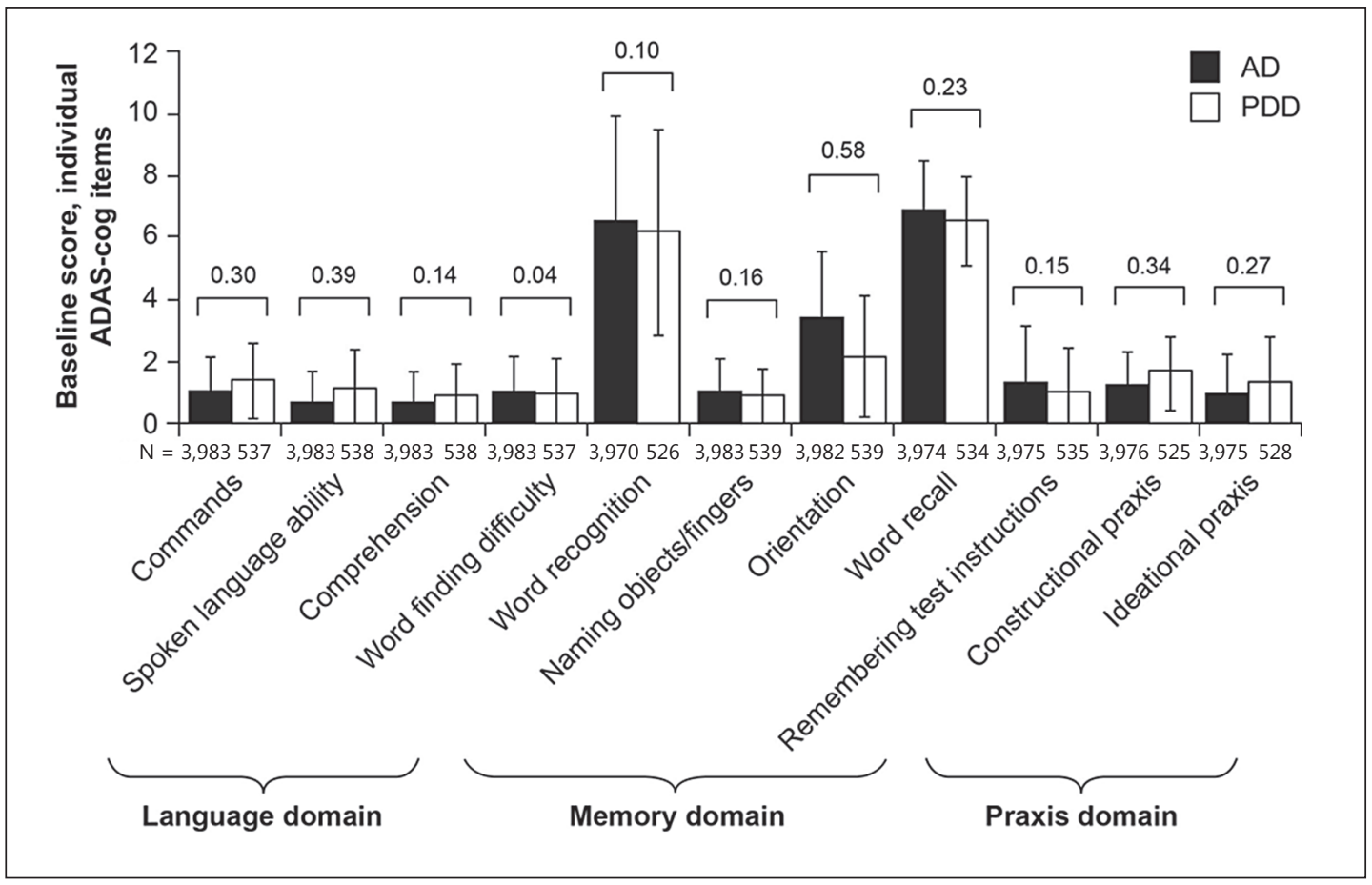

Fig. 2. Baseline scores on the individual ADAS-cog items that comprise the symptom domains of language, memory and praxis for the AD (IDEAL and ADENA) and PDD (EXPRESS) patient populations (randomized population). Values represent the calculated standardized differences comparing the AD and PDD patient populations. Error bars represent the SD from the mean. Standardized differences were calculated using an ANCOVA model with study as a factor and corresponding baseline score as a covariate (where appropriate). Higher scores reflect a greater impairment.

ADCS-CGIC Scores and the Change from Baseline at Week 24/26 on the ADAS-cog, ADCS-ADL and NPI-10 Scales in Patients with AD and PDD Receiving Placebo

Patients with $A D$ in the placebo group showed a greater mean change from baseline at week 24/26 than patients with PDD on the total ADAS-cog (standardized difference $=0.14$ AD vs. PDD; fig. 3a). Similar findings were observed when including disease severity (baseline MMSE score) as a covariate in the ANCOVA model used to compare the AD and PDD patient populations (standardized difference $=0.16 \mathrm{AD}$ vs. PDD). No differences were seen between the patient populations on the ADCS-CGIC, irrespective of the baseline MMSE score [standardized difference $=0.09$ (model 1) and 0.08 (model 2) AD vs. PDD; fig. 3b]. Irrespective of baseline disease severity (MMSE), greater decline from baseline to week 24 was observed on the NPI-10 in patients with PDD compared with those with $\mathrm{AD}$ [standardized difference $=0.11$ (model 1 ) and 0.29 (model 2) AD vs. PDD; fig. 3c]. A trend towards greater worsening was observed on the ADCS-ADL amongst patients with PDD compared with those with AD [standardized difference $=0.08 \mathrm{AD}$ vs. PDD (model 1); fig. 3d]. When taking into account baseline disease severity, patients with PDD randomized to receive placebo showed a greater mean change from baseline at the study endpoint than patients with $\mathrm{AD}$ (standardized difference $=0.35 \mathrm{AD}$ vs. PDD).

Patients with $\mathrm{AD}$ randomized to receive placebo showed a greater mean (SD) change from baseline at week 24/26 than PDD patients who received placebo on the ADAS-cog memory domain $[1.3(4.20)$ and $0.3(4.90)$ for $\mathrm{AD}(\mathrm{n}=1,148)$ and PDD $(\mathrm{n}=158)$, respectively; standardized difference $=0.25$ (model 1$)$ and 0.34 (model 2$)$ AD vs. PDD]. A greater mean change from baseline at the study endpoint on the ADAS-cog praxis domain was also observed 


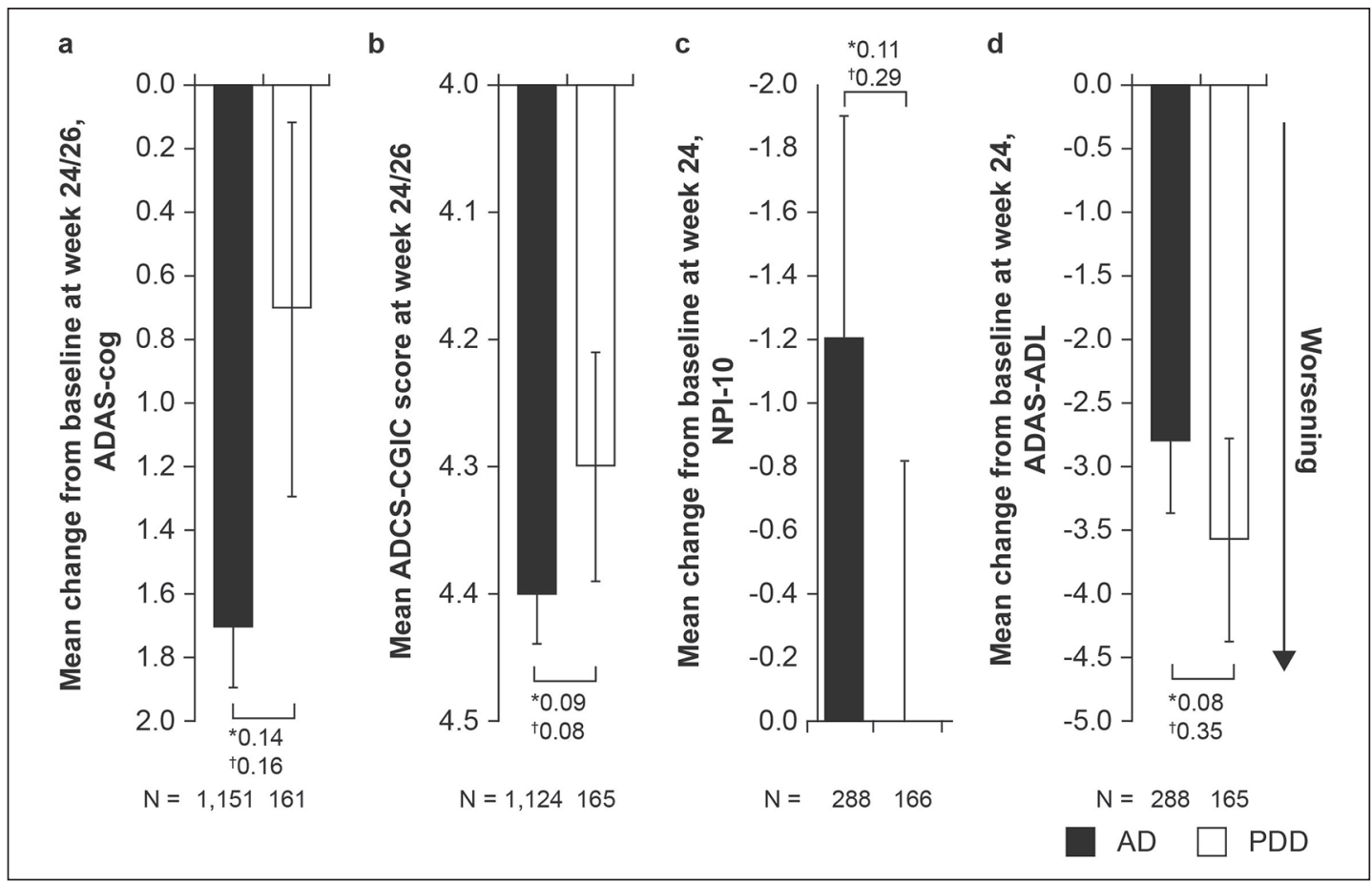

Fig. 3. Mean change from baseline at week 24/26 on ADAS-cog (a), mean ADCS-CGIC score at week 24/26 (b), mean change from baseline at week 24 on NPI-10 (c), and mean change from baseline at week 24 on ADCS-ADL (intent-to-treat population) (d). Standardized differences (Cohen's d) were calculated to compare the ADCS-CGIC score and the change in ADAS-cog, NPI-10 and ADCS-ADL score between the AD and PDD patient populations. * ANCOVA model with study as a factor and corresponding baseline score as a covariate (where appropriate); ${ }^{\dagger}$ ANCOVA model with study as a factor and baseline MMSE score and corresponding baseline score as covariates (where appropriate). ADAS-cog and ADCS-CGIC data were outcome measures in IDEAL, ADENA and EXPRESS. ADCS-ADL and NPI-10 were outcome measures in IDEAL and EXPRESS only.

in patients with AD compared with patients with PDD. However, the observed difference was not maintained when baseline MMSE was included as a covariate in the model used to compare the AD and PDD patient populations [mean (SD) = $0.3(1.48)$ and $0.1(1.83)$ for $\mathrm{AD}(\mathrm{n}=1,148)$ and PDD $(n=152)$, respectively; standardized difference $=0.14$ (model 1 ) and 0.00 (model 2) AD vs. PDD, respectively]. A trend towards a greater change from baseline in the ADAS-cog language domain was observed in patients with $\mathrm{AD}$ compared with patients with PDD [0.6 (2.63) and 0.4 (3.05) for AD $(n=1,152)$ and PDD $(n=161)$, respectively]. However, the calculated standardized difference falls below the applied threshold of 0.1 [standardized difference $=0.07($ model 1$)$ and $0.00($ model 2$)$ AD vs. PDD $)$.

\section{Discussion}

Despite cognitive deficits in both AD and PDD being related to cholinergic deficiency, mounting evidence suggests that these dementias are clinically and biologically distinct entities. PDD is commonly associated with $\alpha$-synuclein (Lewy body) pathology, while AD is associated with neuritic plaques and neurofibrillary tangles in the basal forebrain $[7,24]$. Although there is a clear overlap in the clinical presentation of both diseases, there are also distinct differences. PD is typically classified as a movement disorder; however, there are also 
non-motor features, including cognitive impairment [25]. Research has suggested that brain cholinergic deficiencies may be greater in PDD than in mild AD [26]. Patients with PDD predominantly show deficits in attention, executive function and visual spatial function [27, 28]. Memory impairment, which is dominant in AD, is less prominent in PDD [29]. The predominant language abnormality in PDD is impairment of verbal fluency, which is more marked in PDD compared with AD [7, 30]. Naming difficulties and impaired comprehension are also observed in PDD, although to a lesser extent than in patients with AD [7, 31]. Neuropsychiatric symptoms are common in both AD and PDD [28, 32]. Visual hallucinations, although present in both AD and PDD, tend to arise more commonly in PDD than in AD [33].

In the current analysis, we compared baseline impairment in patients with AD and PDD on a range of outcome measures, namely the ADAS-cog, ADCS-ADL and NPI-10. These data suggest that patients with $\mathrm{AD}$ demonstrate greater impairment in memory than patients with PDD, while patients with PDD tended to show greater impairment in language (except wordfinding difficulty) and praxis. Overall, the greatest difference between patients with AD and PDD was observed on the orientation (memory) item. This observation is consistent with a previous analysis published by Bronnick et al. [20]. Given the impact of PD on motor function, the observed greater impairment of praxis in PDD compared with AD was not unexpected. Bronnick et al. [20] also reported greater impairment of praxis in patients with PDD. However, they also noted that this might be difficult to interpret given the impact of motor disability on a patient's ability to perform tasks, a point important to consider when interpreting the results of the current analysis.

Previous analyses have reported significant differences between patients with $A D$ and PDD on the commands, constructional praxis, ideational praxis, word recall, word recognition and orientation items of the ADAS-cog, and the serial 7s subtraction task from the MMSE [20]. Based on the Z-scores, the groups differed most on attention and calculation (assessed using the MMSE), followed by orientation [20]. A direct logistic regression analysis showed that these variables reliably distinguished patients with AD and PDD with an overall success rate of $74.7 \%$ [20].

In these studies, patients with AD were found to score higher on the ADCS-ADL scale at baseline than patients with PDD, suggesting that patients with PDD are more impaired in their ability to perform ADL. This is not unexpected, as PD is a complex movement disorder, in which symptoms such as rigidity, bradykinesia and tremor can compound cognitive deficits to further restrict a patient's ability to perform tasks. Behavioral disturbances, as assessed using the NPI-10, appeared to manifest at similar levels in the two patient populations. However, stable doses of psychotropic medications were permitted at baseline in IDEAL and EXPRESS, and limited use of select medications in the ADENA program that may have masked behavioral disturbances in these patients.

Deterioration in global function, cognition, ADL and behavior was calculated for patients with AD and PDD randomized to receive placebo in these studies, allowing a comparison of disease progression over time in untreated AD and PDD patient populations. Overall, patients with AD showed greater deterioration in cognition (particularly memory) and fewer behavioral symptoms compared with patients with PDD, but similar levels of decline in ADL and global function were seen for both patient groups. This trend (for cognition and ADL) is consistent with previous studies of rivastigmine in these patient populations [28]. Further analyses taking into account the baseline MMSE score supported these findings, with the exception of the ADCS-ADL scale, where patients with PDD showed a greater mean change from baseline to the study endpoint than patients with AD. This suggests disease severity has a greater impact on deterioration of the ability to perform ADL in patients with PDD compared with AD. These data suggest that despite there being a common cholinergic basis to these diseases, they are distinctly different in aspects of their clinical features and progression. 
Cognitive decline may be slower in PDD although this could be influenced by the range of cognitive domains assessed in these studies, since the ADAS-cog was originally developed to monitor progression in AD. As such, the scales employed may not fully quantify specific cognitive and functional impairments in the PDD patient population.

This was a retrospective, hypothesis-forming, exploratory analysis of baseline and follow-up data collected during the IDEAL study, ADENA program and EXPRESS. Data on AD and PDD patients were derived from three independent trial databases, which were not prospectively powered to detect differences in baseline characteristics or longitudinal differences between the patient populations. As a result, the data should be interpreted with caution. Larger studies enrolling both AD and PDD patients would be required to fully investigate and compare the range of impairments observed in these populations.

The findings of the current analyses demonstrate differing patterns of impairment between patients with AD and PDD, despite both illnesses involving cholinergic deficiencies. The results support previous suggestions that the dementia associated with PD is not simply $\mathrm{AD}$ comorbid to PD. Greater understanding of the clinical features and the progression of these disease states will be valuable in improving the accuracy of diagnosis and appropriate management of these conditions.

\section{Acknowledgements}

The development of the manuscript was supported by Novartis Pharmaceuticals Corporation, East Hanover, N.J., USA. Novartis have developed and manufacture rivastigmine and sponsored the large, multicenter, randomized, double-blind trials that led to the approval of rivastigmine for mild-to-moderate dementia of the Alzheimer's type and dementia associated with PD in the USA. Medical writing and editorial assistance in the preparation of this article were provided by Katy Cooke of Fishawack Communications Ltd, Oxford, UK.

\section{Disclosure Statement}

M.F. has provided expert consultation services and received speaker honoraria and research funding from Novartis Pharmaceuticals Corporation. F.S. has received research funding in the past from Pfizer and Forest Laboratories. D.A. has received research support from H. Lundbeck and Merck-Serono, and honoraria from H. Lundbeck, Novartis and GE Healthcare. G.G. has received personal compensation for consulting and research funding to his academic institute and department from Novartis Pharmaceuticals Corporation. M.S. and X. M. are employees and stock holders of Novartis Pharmaceuticals Corporation.

\section{References}

1 Davies P, Maloney AJ: Selective loss of central cholinergic neurons in Alzheimer's disease. Lancet 1976;2:1403.

-2 Winblad B, Cummings J, Andreasen N, Grossberg G, Onofrj M, Sadowsky C, Zechner S, Nagel J, Lane R: A sixmonth double-blind, randomized, placebo-controlled study of a transdermal patch in Alzheimer's disease rivastigmine patch versus capsule. Int J Geriatr Psychiatry 2007;22:456-467.

3 Rösler M, Anand R, Cicin-Sain A, Gauthier S, Agid Y, Dal-Bianco P, Stahelin HB, Hartman R, Gharabawi M: Efficacy and safety of rivastigmine in patients with Alzheimer's disease: international randomised controlled trial. BMJ 1999;318:633-638.

4 Corey-Bloom J, Anand R, Veach J: A randomized trial evaluating the efficacy and safety of ENA 713 (rivastigmine tartrate), a new acetylcholinesterase inhibitor, in patients with mild to moderately severe Alzheimer's disease. Int J Geriatr Psychopharmacol 1998;1:55-65. 
5 Tiraboschi P, Hansen LA, Alford M, Sabbagh MN, Schoos B, Masliah E, Thal LJ, Corey-Bloom J: Cholinergic dysfunction in diseases with Lewy bodies. Neurology 2000;54:407-411.

6 Braak H, Del Tredici K, Rub U, de Vos RA, Jansen Steur EN, Braak E: Staging of brain pathology related to sporadic Parkinson's disease. Neurobiol Aging 2003;24:197-211.

7 Emre M: Dementia associated with Parkinson's disease. Lancet Neurol 2003;2:229-237.

8 Aarsland D, Zaccai J, Brayne C: A systematic review of prevalence studies of dementia in Parkinson's disease. Mov Disord 2005;20:1255-1263.

$\$ 9$ Emre M, Aarsland D, Albanese A, Byrne EJ, Deuschl G, De Deyn PP, Durif F, Kulisevsky J, van Laar T, Lees A, Poewe W, Robillard A, Rosa MM, Wolters E, Quarg P, Tekin S, Lane R: Rivastigmine for dementia associated with Parkinson's disease. N Engl J Med 2004;351:2509-2518.

10 Rosen WG, Mohs RC, Davis KL: A new rating scale for Alzheimer’s disease. Am J Psychiatry 1984;141:13561364.

11 Weyer G, Erzigkeit H, Kanowski S, Ihl R, Hadler D: Alzheimer's disease assessment scale: reliability and validity in a multicenter clinical trial. Int Psychogeriatr 1997;9:123-138.

12 Harvey PD, Ferris SH, Cummings JL, Wesnes KA, Hsu C, Lane RM, Tekin S: Evaluation of dementia rating scales in Parkinson's disease dementia. Am J Alzheimers Dis Other Demen 2010;25:142-148.

13 Talwalker S, Overall JE, Srirama MK, Gracon SI: Cardinal features of cognitive dysfunction in Alzheimer's disease: a factor-analytic study of the Alzheimer's Disease Assessment Scale. J Geriatr Psychiatry Neurol 1996; 9:39-46.

14 Olin J, Schneider L: Assessing response to tacrine using the factor analytic structure of the Alzheimer's Disease Assessment Scale (ADAS) - cognitive subscale. Int J Geriatr Psychiatry 1995;10:753-756.

15 Feldman HH, Lane R: Rivastigmine: a placebo-controlled trial of bid and tid regimens in patients with Alzheimer's disease. J Neurol Neurosurg Psychiatry 2007;78:1056-1063.

16 Schneider LS, Anand R, Farlow MR: Systematic review of the efficacy of rivastigmine for patients with Alzheimer's disease. Int J Geriatr Psychopharmacol 1998;1:S26-S34.

17 Galasko D, Bennett D, Sano M, Ernesto C, Thomas R, Grundman M, Ferris S: An inventory to assess activities of daily living for clinical trials in Alzheimer's disease. The Alzheimer's Disease Cooperative Study. Alzheimer Dis Assoc Disord 1997;11(suppl 2):S33-S39.

18 Cummings JL, Mega M, Gray K, Rosenberg-Thompson S, Carusi DA, Gornbein J: The neuropsychiatric inventory: comprehensive assessment of psychopathology in dementia. Neurology 1994;44:2308-2314.

19 Schneider LS, Olin JT, Doody RS, Clark CM, Morris JC, Reisberg B, Schmitt FA, Grundman M, Thomas RG, Ferris SH: Validity and reliability of the Alzheimer's disease cooperative study-clinical global impression of change. The Alzheimer's Disease Cooperative Study. Alzheimer Dis Assoc Disord 1997;11(suppl 2):S22-S32.

20 Bronnick K, Emre M, Lane R, Tekin S, Aarsland D: Profile of cognitive impairment in dementia associated with Parkinson's disease compared with Alzheimer's disease. J Neurol Neurosurg Psychiatry 2007;78:1064-1068.

21 Diagnostic and Statistical Manual of Mental Disorders (DSM-IV), ed 4. Arlington, American Psychiatric Association, 1994.

-22 McKhann G, Drachman D, Folstein M, Katzman R, Price D, Stadlan EM: Clinical diagnosis of Alzheimer disease: report of the NINCDS-ADRDA Work Group under the auspices of Department of Health and Human Services Task Force on Alzheimer's Disease. Neurology 1984;34:939-944.

-23 Folstein MF, Folstein SE, McHugh PR: 'Mini-mental state'. A practical method for grading the cognitive state of patients for the clinician. J Psychiatr Res 1975;12:189-198.

24 Jellinger K: The pedunculopontine nucleus in Parkinson's disease, progressive supranuclear palsy and Alzheimer's disease. J Neurol Neurosurg Psychiatry 1988;51:540-543.

25 Bohnen NI, Albin RL: The cholinergic system and Parkinson disease. Behav Brain Res 2011;221:564-573.

-26 Bohnen NI, Kaufer DI, Ivanco LS, Lopresti B, Koeppe RA, Davis JG, Mathis CA, Moore RY, DeKosky ST: Cortical cholinergic function is more severely affected in parkinsonian dementia than in Alzheimer disease: an in vivo positron emission tomographic study. Arch Neurol 2003;60:1745-1748.

27 Galvin JE, Pollack J, Morris JC: Clinical phenotype of Parkinson disease dementia. Neurology 2006;67:16051611.

28 Emre M, Cummings JL, Lane RM: Rivastigmine in dementia associated with Parkinson's disease and Alzheimer's disease: similarities and differences. J Alzheimers Dis 2007;11:509-519.

29 Helkala EL, Laulumaa V, Soininen H, Riekkinen PJ: Recall and recognition memory in patients with Alzheimer's and Parkinson's diseases. Ann Neurol 1988;24:214-217.

-30 Stern Y, Richards M, Sano M, Mayeux R: Comparison of cognitive changes in patients with Alzheimer's and Parkinson's disease. Arch Neurol 1993;50:1040-1045.

-31 Cummings JL, Darkins A, Mendez M, Hill MA, Benson DF: Alzheimer's disease and Parkinson's disease: Comparison of speech and language alterations. Neurology 1988;38:680-684.

-32 Aarsland D, Cummings JL, Larsen JP: Neuropsychiatric differences between Parkinson's disease with dementia and Alzheimer's disease. Int J Geriatr Psychiatry 2001;16:184-191.

-33 Farlow M, Cummings J: A modern hypothesis: the distinct pathologies of dementia associated with Parkinson's disease versus Alzheimer's disease. Dement Geriatr Cogn Disord 2008;25:301-308. 